\title{
Papers
}

\section{Cost effectiveness analysis of laparoscopic hysterectomy compared with standard hysterectomy: results from a randomised trial}

\author{
Mark Sculpher, Andrea Manca, Jason Abbott, Jayne Fountain, Su Mason, Ray Garry
}

\begin{abstract}
Objective To assess the cost effectiveness of laparoscopic hysterectomy compared with conventional hysterectomy (abdominal or vaginal).

Design Cost effectiveness analysis based on two parallel trials: laparoscopic $(\mathrm{n}=324)$ compared with vaginal hysterectomy $(\mathrm{n}=163)$; and laparoscopic $(\mathrm{n}=573)$ compared with abdominal hysterectomy $(\mathrm{n}=286)$.

Participants 1346 women requiring a hysterectomy for reasons other than malignancy.

Main outcome measure One year costs estimated from NHS perspective. Health outcomes expressed in terms of QALYs based on women's responses to the EQ-5D at baseline and at three points during up to 52 weeks' follow up.

Results Laparoscopic hysterectomy cost an average of $£ 401$ ( $\$ 708$; €571) more (95\% confidence interval £271 to £542) than vaginal hysterectomy but produced little difference in mean QALYs $(0.0015,-0.015$ to 0.018$)$. Mean differences in cost and QALYs generated an incremental cost per QALY gained of $£ 267333$ (\$471 789; €380 437). The probability that

laparoscopic hysterectomy is cost effective was below $50 \%$ for a large range of values of willingness to pay for an additional QALY. Laparoscopic hysterectomy cost an average of $£ 186$ (\$328; €265) more than abdominal hysterectomy, although 95\% confidence intervals crossed zero ( $-£ 26$ to $£ 375$ ); there was little difference in mean QALYs $(0.007,-0.008$ to 0.023$)$, resulting in an incremental cost per QALY gained of £26 571 (\$46 893; €37 813). If the NHS is willing to pay $£ 30000$ for an additional QALY, the probability that laparoscopic hysterectomy is cost effective is $56 \%$.

Conclusions Laparoscopic hysterectomy is not cost effective relative to vaginal hysterectomy. Its cost effectiveness relative to the abdominal procedure is finely balanced.
\end{abstract}

\section{Introduction}

Hysterectomy is a common operation, with up to $100000^{1}$ and $550000^{2}$ procedures undertaken annually in the United Kingdom and the United States, respectively. Traditionally, most hysterectomies have been undertaken through the abdomen, but there have been no randomised comparisons of abdominal and vaginal hysterectomy. The advent of laparoscopic approaches to hysterectomy offers the prospect of improved outcomes and gains in cost effectiveness through reduced severity of convalescence and shorter length of inpatient stay. With the exception of some observational studies ${ }^{3-5}$ and small randomised trials, ${ }^{67}$ however, little is known about the costs and cost effectiveness of laparoscopic forms of hysterectomy relative to conventional (abdominal and vaginal) approaches.

The eVALuate trial is the largest trial of laparoscopic hysterectomy compared with standard methods yet undertaken. ${ }^{8}$ This report describes a cost effectiveness analysis undertaken with eVALuate data.

\section{Methods}

Overview

Over one year we estimated costs from the NHS perspective and expressed benefits in terms of quality adjusted life years (QALYs). We undertook two separate comparisons: laparoscopic hysterectomy (ALH) versus abdominal and laparoscopic hysterectomy (VLH) versus vaginal hysterectomy.

\section{Trial design}

Full details of the design of the eVALuate trial are reported in the accompanying paper. ${ }^{8}$ All the women we randomised had gynaecological symptoms that indicated the need for a hysterectomy. We excluded women with confirmed or suspected malignant disease of the genital tract. Once a woman was included, the surgeon decided which form of conventional hysterectomy was most appropriate, abdominal or vaginal. Women were then randomised between the selected conventional procedure and laparoscopic procedure in two parallel trials: vaginal versus laparoscopic hysterectomy and abdominal versus laparoscopic hysterectomy. We used a 2:1 randomisation schedule in favour of the laparoscopy.

Of the 1380 women who were randomised into the study, 34 withdrew before surgery was undertaken. Of the 859 women who were allocated to and received treatment in the abdominal part of the study, 573 were randomised to laparoscopic hysterectomy and 286 to abdominal hysterectomy. Of the 487 who were allocated to and received treatment in the vaginal part of the trial, 324 patients were randomised to laparoscopic hysterectomy and 163 to vaginal hysterectomy. We carried out the economic analysis on data from the 1346 women who did not drop out before surgery over a median follow up of 52 weeks (range 6-52; mean 46.88).

\section{Measurement of resource use}

For purposes of costing we collected data on the use of resources under several headings.

Theatre-Clinical staff completed case record forms on the use of theatre resources. This included time in theatre and recovery room; type of hysterectomy undertaken; use of prophylactic antibiotics and anticoagulants; type of anaesthetic; method of haemostasis; and use of specific consumables such as disposable 


\begin{tabular}{|c|c|c|c|}
\hline Item of resource & Unit & Unit cost $(£)$ & Source \\
\hline \multicolumn{4}{|l|}{ Ward: } \\
\hline General ward & Day & 135.50 & $\begin{array}{c}\text { Mean of two specific } \\
\text { hospitals recruiting to } \\
\text { eVALuate }\end{array}$ \\
\hline High dependency unit & Day & 393.66 & Sculpher et al ${ }^{9}$ \\
\hline Intensive care unit & Day & 866.83 & Sculpher et al ${ }^{9}$ \\
\hline \multicolumn{4}{|l|}{ Theatre: } \\
\hline Staff (variable) & Minute & 2.26 & Booth $^{10}$ Gough $^{11}$ \\
\hline Staff (fixed) & Fixed & 1.36 & Booth $^{10}$ Gough $^{11}$ \\
\hline Overheads & Minute & 1.83 & $\begin{array}{c}\text { Mean of two specific } \\
\text { hospitals recruiting to } \\
\text { eVALuate }\end{array}$ \\
\hline \multicolumn{4}{|c|}{ Selected consumables used during laparoscopic assisted procedure: } \\
\hline Linear stapler & item & 257.72 & Manufacturer \\
\hline Laparoscopic scissors & item & 120.44 & Manufacturer \\
\hline Disposable trocars & item & 76.57 & Manufacturer \\
\hline \multicolumn{4}{|l|}{ Visits: } \\
\hline GP visits & Visit & 15.75 & Netten et $\mathrm{al}^{12}$ \\
\hline $\begin{array}{l}\text { Outpatient hospital } \\
\text { visits }\end{array}$ & Visit & 62.00 & $\mathrm{CIPFA}^{13}$ \\
\hline Day care visits & Visit & 62.00 & $\begin{array}{c}\text { Assumed the same as } \\
\text { outpatient visit }\end{array}$ \\
\hline
\end{tabular}

trocars and scissors. Details of intraoperative complications were also collected, including the need for blood transfusion. JA, who was blinded to treatment allocation, estimated any additional use of resources associated with these complications (such as drugs or tests).

Main admission to hospital-Case record forms were also used to measure use of other resources during a woman's main admission, including total length of stay in hospital (by type of ward) and the use of urinary catheterisation. We also collected details of postoperative complications during admission, including any blood transfusion and whether a woman had to be returned to theatre; additional resource use was estimated as for operative complications.

Follow up-At the six week clinic follow up visit, we used case record forms to collect data on the incidence of any complications; any additional resource use was estimated as for the immediate postoperative period. Patients also completed a questionnaire at this point, which included questions on number of inpatient days and outpatient, day case, and general practice visits made for any reason after they left hospital. Patients were also asked to complete similar questionnaires 4 and 12 months after hospital discharge.

\section{Unit costs}

We used UK unit costs at 1999-2000 prices to value the use of resources. Table 1 details the key unit costs, together with their sources. We costed any inpatient days in hospital after the main admission using average inpatient costs per day from English hospitals. $^{13}$

\section{Health outcomes}

The health outcomes of the alternative forms of hysterectomy were assessed in terms of quality adjusted life years (QALYs). This reflects any mortality and differences in health-related quality of life based on women's responses to the EQ-5D questionnaire at baseline and at up to three points after hospital discharge (six weeks, four months, and one year). The EQ-5D is a generic measure of health status, where health is characterised on five dimensions (mobility, self care, ability to undertake usual activities, pain, anxiety/depression).$^{14}$ At each point of follow up, women were asked to indicate their health on each dimension using one of three levels: no problems, moderate problems, and severe problems. Each response placed a woman into one of 245 mutually exclusive health states, and a value for each of these has previously been estimated on the 0 (equivalent to dead) to 1 (equivalent to good health) "utility" scale based on interviews with a sample of 3395 members of the UK public. ${ }^{15}$ Hence, each woman in the trial had a health utility score at up to four time points and, by using area under the curve methods ${ }^{16}$ which effectively multiplies utility by time, we translated these observations into QALYs over each woman's period of follow up. In estimating mean QALYs in each group, we used regression methods to adjust for differences in baseline EQ-5D utility.

\section{Analysis}

We used STATA (release 6.0, College Station TX) for statistical analyses. As a result of staggered entry into the trial, 182 women were not followed up for a full year. We therefore estimated mean costs and QALYs over one year by using methods to adjust for censored data. ${ }^{18}$ Given the short time horizon, costs and QALYs remain undiscounted. To account for the skewed nature of the data, we calculated $95 \%$ confidence intervals for differential costs and QALYs using the bias corrected and accelerated bootstrap method. ${ }^{19}{ }^{20}$ Cost effectiveness analysis was undertaken to relate differential mean costs and QALYs associated with the alternative arms of the trial, with incremental cost effectiveness ratios (ICERs) calculated as appropriate. To account for uncertainty due to sampling variation in cost effectiveness, we plotted cost effectiveness acceptability curves, ${ }^{21}$ showing the probability of laparoscopic hysterectomy being more cost effective than conventional hysterectomy for different maximum levels that the decision maker may be willing to pay for an additional QALY.

\section{Results}

\section{Resource use}

Table 2 provides a summary of the key measures of resource use in the trial; results are presented separately for the two comparisons in the study. For the comparison of laparoscopic and vaginal hysterectomy, the main differences related to time in theatre (mean $98 v 65$ minutes, respectively) and the use of disposable equipment in many laparoscopic hysterectomies-for example, a disposable linear stapler was used to achieve haemostasis in 36\% of ovarian pedicles and 19\% of uterine pedicles, and disposable scissors were used in $37 \%$ of laparoscopic hysterectomies (more details are available elsewhere $\left.{ }^{22}\right)$. No marked differences emerged between the procedures in length of stay or use of resources after the initial admission.

The second comparison, between laparoscopic and abdominal hysterectomy, showed rather more differences in terms of use of resources (table 2). Again, time in theatre was longer with laparoscopic hysterectomy (mean 108 v 74 minutes). Also, a high proportion of laparoscopic procedures used disposable equipment. Compared with abdominal hysterectomy, however, laparoscopic hysterectomy had a lower mean length of hospital stay (3.95 $v 5.11$ days). During follow up, there were no differences in use of resources that would be expected to have a large effect on differential cost.

\section{Costs}

Table 3 shows mean and median costs per patient. For the comparison of laparoscopic and vaginal hysterectomy, the only marked difference related to theatre cost, which reflects differences in theatre times and the use of disposable equipment in a large proportion of laparoscopic procedures. None of the 
Table 2 Key resource use measured in two parts of trial comparing different methods of hysterectomy. Figures are numbers (percentages) of patients unless stated otherwise

\begin{tabular}{|c|c|c|c|c|c|c|c|c|}
\hline \multirow[b]{2}{*}{ Item of resource use } & \multicolumn{4}{|c|}{ Vaginal trial } & \multicolumn{4}{|c|}{ Abdominal trial } \\
\hline & \multicolumn{2}{|c|}{ Laparoscopy $(\mathrm{n}=324)$} & \multicolumn{2}{|c|}{ Vaginal $(n=163)$} & \multicolumn{2}{|c|}{ Laparoscopy $(n=573)$} & \multicolumn{2}{|c|}{ Abdominal $(\mathrm{n}=286)$} \\
\hline \multicolumn{9}{|c|}{ Main admission to hospital } \\
\hline \multicolumn{9}{|c|}{ Time in theatre (minutes) ${ }^{\star}$ : } \\
\hline Mean (SD) & 98.14 & $(35.45)$ & 65.03 & $(27.87)$ & 108.07 & (33.33) & 74.08 & $(23.86)$ \\
\hline Median (IQR) & \multicolumn{2}{|c|}{$95 \quad(73-120)$} & \multicolumn{2}{|c|}{$58 \quad(47-75)$} & \multicolumn{2}{|c|}{$105 \quad(89-125)$} & \multicolumn{2}{|c|}{$70 \quad(60-85)$} \\
\hline \multicolumn{9}{|c|}{ Total length of stay in hospital (days)†: } \\
\hline Mean (SD) & 4.28 & $(2.02)$ & 4.32 & $(1.99)$ & 3.95 & $(2.36)$ & 5.11 & $(2.70)$ \\
\hline Median (IQR) & 4 & $(3-5)$ & 4 & $(3-5)$ & 4 & $(3-5)$ & 5 & $(4-6)$ \\
\hline \multicolumn{9}{|l|}{ Six week follow up } \\
\hline \multicolumn{9}{|l|}{ Outpatient visits: } \\
\hline 0 & 173 & (77) & 83 & (70) & 329 & (72) & 143 & (67) \\
\hline 1 & 42 & (19) & 34 & (29) & 104 & (23) & 62 & (29) \\
\hline$>1$ & 11 & (5) & 2 & (2) & 24 & (5) & 10 & (5) \\
\hline \multicolumn{9}{|l|}{ GP visits: } \\
\hline 0 & 53 & $(24)$ & 32 & (37) & 103 & (23) & 47 & $(22)$ \\
\hline $1-5$ & 169 & (75) & 84 & (71) & 349 & (77) & 161 & (75) \\
\hline$>5$ & 4 & (2) & 2 & (17) & 5 & (1) & 7 & (3) \\
\hline \multicolumn{9}{|l|}{ Inpatient visits: } \\
\hline 0 & 217 & $(96)$ & 113 & (96) & 426 & (93) & 205 & (95) \\
\hline 1 & 6 & (3) & 5 & (4) & 28 & (6) & 10 & (5) \\
\hline 2 & 3 & (1) & - & & 3 & (1) & - & \\
\hline
\end{tabular}

Four month follow up

Outpatient visits:

\begin{tabular}{|c|c|c|c|c|c|c|c|c|}
\hline 0 & 143 & (75) & 83 & (81) & 289 & (75) & 119 & (70) \\
\hline 1 & 36 & (19) & 14 & (14) & 76 & (20) & 35 & (21) \\
\hline$>1$ & 13 & (7) & 5 & (5) & 20 & (5) & 15 & (9) \\
\hline \multicolumn{9}{|c|}{ GP visits: } \\
\hline 0 & 71 & (37) & 59 & (58) & 116 & (30) & 52 & (31) \\
\hline $1-5$ & 115 & (60) & 60 & (59) & 255 & (66) & 113 & (67) \\
\hline$>5$ & 6 & $(0.03)$ & 3 & $(0.03)$ & 14 & $(0.03)$ & 4 & $(0.02)$ \\
\hline \multicolumn{9}{|c|}{ Inpatient visits: } \\
\hline 0 & 189 & (98) & 100 & (98) & 381 & (99) & 162 & (96) \\
\hline 1 & 3 & (1) & 2 & (2) & 3 & (1) & 7 & (4) \\
\hline$>1$ & - & & - & & 1 & $(0.01)$ & - & \\
\hline
\end{tabular}

\section{One year follow up}

Outpatient visits:

\begin{tabular}{|c|c|c|c|c|c|c|c|c|}
\hline 0 & 177 & (81) & 87 & (77) & 313 & (75) & 143 & (73) \\
\hline 1 & 26 & (12) & 18 & (18) & 60 & (14) & 28 & (15) \\
\hline$>1$ & 15 & (7) & 8 & (8) & 45 & (11) & 17 & (4) \\
\hline \multicolumn{9}{|c|}{ GP visits: } \\
\hline 0 & 55 & (25) & 26 & (23) & 95 & (23) & 47 & (25) \\
\hline $1-5$ & 131 & (60) & 72 & (64) & 259 & (62) & 117 & (62) \\
\hline$>5$ & 32 & (15) & 15 & (13) & 64 & (15) & 24 & (13) \\
\hline \multicolumn{9}{|c|}{ Inpatient visits: } \\
\hline 0 & 213 & (98) & 104 & (92) & 402 & (96) & 177 & (94) \\
\hline$>0$ & 5 & (2) & 9 & (8) & 16 & (4) & 11 & (6) \\
\hline
\end{tabular}

IQR=interquartile range

*Calculated as patient's preparation time plus time from first incision to last suture.

†Calculated as discharge date minus admission date.

other cost components showed marked differences between the groups. Overall, laparoscopic hysterectomy cost a mean of $£ 401$ (95\% confidence interval £271 to £542) more per patient.

The comparison of laparoscopic with abdominal hysterectomy showed that costs for laparoscopy were closer to, but still higher than for, conventional hysterectomy. A mean difference of $£ 335$ in theatre costs again reflects longer theatre times and the use of disposable equipment with laparoscopy. However, the shorter length of admission with laparoscopic hysterectomy offset some of that additional cost, with a mean saving in hotel costs of $£ 144$. Overall, laparoscopic hysterectomy cost a mean of $£ 186$ more per patient, with $95 \%$ confidence intervals crossing zero ( $-£ 26$ to $£ 375)$.

\section{Health outcomes}

There were no deaths during follow up. In terms of both mean and median EQ-5D values, and for both comparisons, women showed improvements between baseline and six weeks and between six weeks and four months; and little change between four months and a year (table 4). The utilities were used to calculate QALYs for each woman over a one year period (table 4). These differences were small and 95\% confidence intervals crossed zero. Mean QALYs per patient were higher with laparoscopic hysterectomy, both compared with vaginal hysterectomy $(0.0015,-0.015$ to 0.018$)$ and the abdominal procedure $(0.007$, -0.008 to 0.023$)$. 
Table 3 Comparison of costs between laparoscopic and standard hysterectomy (1999-2000 prices)

\begin{tabular}{|c|c|c|c|c|c|c|c|c|}
\hline & \multicolumn{4}{|c|}{ Vaginal trial } & \multicolumn{4}{|c|}{ Abdominal trial } \\
\hline & \multicolumn{2}{|c|}{ Laparoscopy (n=324) } & \multicolumn{2}{|c|}{ Vaginal $(n=163)$} & \multicolumn{2}{|c|}{ Laparoscopy (n=573) } & \multicolumn{2}{|c|}{ Abdominal $(\mathrm{n}=286)$} \\
\hline & Mean & Median (IQR) & Mean & Median (IQR) & Mean & Median (IQR) & Mean & Median (IQR) \\
\hline Theatre cost & 807 & $635(513-919)$ & 396 & $362(309-420)$ & 788 & $646(523-890)$ & 453 & 431 (381-489) \\
\hline Hospital "hotel" cost & 589 & $542(407-678)$ & 591 & $542(407-678)$ & 548 & $542(407-678)$ & 692 & $678(542-813)$ \\
\hline Other postoperative cost & 14 & $(0-0)$ & 18 & $(0-0)$ & 21 & $(0-0)$ & 13 & $(0-0)$ \\
\hline Follow up cost at six weeks & 144 & $46 \quad(0-108)$ & 89 & $46 \quad(0-108)$ & 193 & $46 \quad(0-108)$ & 128 & $(0-108)$ \\
\hline $\begin{array}{l}\text { Follow up cost at four } \\
\text { months }\end{array}$ & 37 & $\begin{array}{ll}0 & (0-46)\end{array}$ & 47 & $\begin{array}{ll}0 & (0-46)\end{array}$ & 39 & $\begin{array}{ll}0 & (0-46)\end{array}$ & 88 & $(0-46)$ \\
\hline Follow up cost at one year & 64 & $(0-46)$ & 112 & $(0-46)$ & 115 & $(0-46)$ & 146 & $(0-46)$ \\
\hline Total cost & 1654 & & 1253 & & 1706 & & 1520 & \\
\hline $\begin{array}{l}\text { Differential mean cost* }(95 \% \\
\mathrm{Cl}) \dagger\end{array}$ & & 271 to 542$)$ & & & & 6 to 375$)$ & & \\
\hline
\end{tabular}

$\mathrm{IQR}=$ interquartile range.

*Laparoscopic minus standard.

† $95 \%$ non-parametric confidence interval based on 1000 bootstrap replications.

Table 4 Health outcomes measured in trial comparing different methods of hysterectomy: responses to EQ-5D and quality adjusted life years (QALYs)

\begin{tabular}{|c|c|c|c|c|c|c|c|c|}
\hline & \multicolumn{4}{|c|}{ Vaginal trial } & \multicolumn{4}{|c|}{ Abdominal trial } \\
\hline & \multicolumn{2}{|c|}{ Laparoscopy (n=324) } & \multicolumn{2}{|c|}{ Vaginal $(n=163)$} & \multicolumn{2}{|c|}{ Laparoscopy $(n=573)$} & \multicolumn{2}{|c|}{ Abdominal $(\mathrm{n}=286)$} \\
\hline & Mean & Median (IQR) & Mean & Median (IQR) & Mean & Median (IQR) & Mean & Median (IQR) \\
\hline \multicolumn{9}{|l|}{ EQ-5D utilities } \\
\hline Baseline & 0.746 & $0.760(0.725-1)$ & 0.758 & $0.796(0.691-1)$ & 0.716 & $0.760(0.691-0.848)$ & 0.690 & $0.725(0.689-0.812)$ \\
\hline Six weeks & 0.875 & $0.907(0.812-1)$ & 0.852 & $0.863(0.76-1)$ & 0.832 & $0.869(0.76-1)$ & 0.833 & $0.883(0.76-1)$ \\
\hline Four months & 0.911 & $0.971(0.848-1)$ & 0.918 & $0.959(0.848-1)$ & 0.886 & $0.959(0.812-1)$ & 0.866 & $0.888(0.796-1)$ \\
\hline One year & 0.920 & $(0.881-1)$ & 0.917 & $(0.861-1)$ & 0.897 & $0.929(0.848-1)$ & 0.892 & $0.959(0.822-1)$ \\
\hline QALYs over one year* & 0.899 & & 0.897 & & 0.870 & & 0.862 & \\
\hline $\begin{array}{l}\text { Differential QALYs over } \\
\text { one yeart }(95 \% \mathrm{Cl}) \neq\end{array}$ & \multicolumn{2}{|c|}{$0.0015(-0.015$ to 0.018$)$} & & & \multicolumn{2}{|c|}{$0.007(-0.008$ to 0.023$)$} & & \\
\hline
\end{tabular}

one yeart $(95 \% \mathrm{Cl})$

*Adjusting for baseline EQ-5D utility.

†Laparoscopic minus standard.

$\$ 95 \%$ non-parametric confidence interval based on 1000 bootstrap replications.

\section{Cost effectiveness}

For the comparison of laparoscopic and vaginal hysterectomy, mean costs were $£ 401$ higher and mean QALYs 0.0015 higher with laparoscopy. In this circumstance, the issue is whether decision makers are willing to pay the implied ICER - that is, the mean difference in cost divided by the mean difference in QALYs-here $£ 267333$ (£401/0.0015). However, we estimated mean differences in costs and QALYs with sampling uncertainty, which is represented in the figure in the form of cost effectiveness acceptability curves. This shows the probability that laparoscopic hysterectomy is more cost effective than vaginal hysterectomy for a range of maximum values that decision makers may place on an additional QALY. The probability that laparoscopic hysterectomy is the more cost effective is never above $50 \%$.

For the comparison of laparoscopic hysterectomy and abdominal hysterectomy, tables 3 and 4 show that laparoscopy had higher mean costs (£186) and higher mean QALYs (0.007) per patient. This generates an ICER of $£ 26571$. The figure shows the cost effectiveness acceptability curve for this comparison, reflecting the imprecision with which these mean differences are estimated. This indicates that the higher the value decision makers place on an additional QALY, the higher the probability that laparoscopic hysterectomy will be more cost effective than abdominal hysterectomy. For example, at a maximum value of $£ 30000$ the probability reaches $56 \%$.

\section{Sensitivity analysis}

We conducted a sensitivity analysis to assess how differential costs would have changed if all laparoscopic procedures had been undertaken with reusable equipment (reusable scissors, sutures rather than staples, and reusable trocars). We assumed

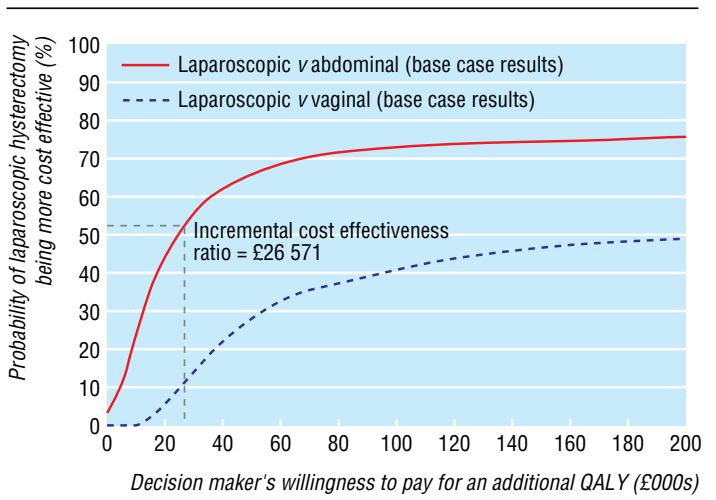

Cost effectiveness acceptability curves for laparoscopic hysterectomy $v$ conventional hysterectomy (abdominal or vaginal). ICER (incremental cost effectiveness ratio) for laparoscopic hysterectomy is not shown as it exceeds $£ 200000$

that there would be no impact on health outcomes from this change in policy. The mean difference in cost between laparoscopic and vaginal hysterectomy was reduced to $£ 260$ and the incremental cost effectiveness ratio for laparoscopy fell to $£ 173$ 334. For the comparison with abdominal hysterectomy, the equivalent figures were $£ 74$ and $£ 10571$. If most of the surgical equipment was disposable the incremental cost effectiveness ratios were $£ 1320667$ for laparoscopic versus vaginal hysterectomy and £259 428 for laparoscopic versus abdominal hysterectomy. Details of further sensitivity analysis are available elsewhere. $^{22}$ 


\section{Discussion}

We have shown that the mean cost of laparoscopic hysterectomy is higher than that of standard hysterectomy, mainly due to the additional cost of disposable instruments used in the procedure. Differences in QALYs between laparoscopic and standard hysterectomy over one year were small. If we focus on mean differences in costs and QALYs, compared with vaginal hysterectomy laparoscopy is unlikely to be considered cost effective as the additional cost associated with generating extra benefit is much higher than the NHS has been willing to pay in other contexts. ${ }^{23}$

The cost effectiveness compared with abdominal hysterectomy is more finely balanced, mainly because of the shorter mean inpatient stay associated with laparoscopy, which results in lower additional costs. The incremental cost of laparoscopy per additional QALY of $£ 26571$ is towards the top of the range that the National Institute for Clinical Excellence has shown itself willing to accept for healthcare interventions in the NHS. ${ }^{23}$ Furthermore, the mean differences in cost and QALYs are measured imprecisely. Reflecting this, the probability of laparoscopy being more cost effective than abdominal hysterectomy is $56 \%$ if the NHS is willing to pay up to $£ 30000$ for an additional QALY, indicating that the decision about value for money is finely balanced. If surgeons use largely reusable equipment in preference to relatively expensive disposables, the additional cost of laparoscopic compared with abdominal hysterectomy would fall to $£ 74$ and the incremental cost effectiveness ratio to $£ 10571$. Although this is more likely to be considered good value for money, this sensitivity analysis should be interpreted with caution as it assumes that the greater use of reusable equipment would not affect outcomes.

\section{Limitations}

A limitation of our study is that health outcomes were not measured until six weeks after the women were discharged from hospital. This may have missed some of the health gains associated with any reduction in the severity of convalescence associated with the laparoscopic procedure. However, differences in utility over a six week period would have a limited effect on QALYs. The absence of utility data during that period is unlikely to change the probable conclusion that laparoscopic rather than vaginal hysterectomy is not cost effective. Given that the cost effectiveness in the comparison with abdominal hysterectomy is more finely balanced, more accurate assessment of the effect of convalescence on utility may be more important in this group.

We used a health service perspective to estimate the costs of the alternative procedures. Any differential impact of the procedures on time away from usual activities, including paid employment, might be reflected in differential productivity costs. The issue of whether productivity costs should be included in cost effectiveness analysis and, if so, how, is a source of controversy. ${ }^{24}$ The eVALuate trial collected data on time away from paid work. These showed that the mean (SD) number of days it took women to return to work after laparoscopic hysterectomy (78.68, SD 44.2) was similar to that in patients undergoing the vaginal procedure (70.21, SD 34.4), and this would not have altered the relative cost effectiveness of these two procedures. However, in the other comparison, women who underwent laparoscopic hysterectomy took fewer days off work than women who underwent the abdominal procedure (77.8 (39.5) v $94.87(60.0))$. If all or part of this difference can reasonably be reflected in terms of productivity savings in monetary terms, this would strengthen
What is already known on this topic?

Hysterectomy is one of the most widely undertaken procedures in the healthcare systems of developed countries

Laparoscopic hysterectomy is being used as an alternative to conventional (abdominal or vaginal) hysterectomy

The differential cost of the conventional and laparoscopic procedures has been assessed only in observational studies and small trials

\section{What this study adds}

Laparoscopic hysterectomy is more costly than conventional hysterectomy, though additional costs are lower in comparison with abdominal than with vaginal hysterectomy

The laparoscopic procedure has a small beneficial effect in terms of quality adjusted life years (QALYs)

Laparoscopic hysterectomy is unlikely to be considered cost effective relative to vaginal hysterectomy. Its cost effectiveness relative to the abdominal procedure is finely balanced

the case for laparoscopic hysterectomy to be considered more cost effective than abdominal hysterectomy.

In conclusion, laparoscopic hysterectomy is unlikely to be considered cost effective relative to vaginal hysterectomy. Its cost effectiveness relative to the abdominal procedure is finely balanced.

We gratefully acknowledge the help of financial staff at eVALuate hospitals in providing cost data.

Contributors: MS designed and supervised the economic analysis and took the lead in drafting the paper. AM undertook the economic analysis and contributed to the drafting of the paper.JA contributed to the cost analysis and to the drafting of the paper. JF contributed to data management and analysis and drafting of the paper. SM supervised the conduct of the trial and contributed to the drafting of the paper. RG led the overall trial and contributed to the drafting of the paper. MS is the guarantor.

Funding: NHS Research and Development Health Technology Assessment Programme. MS has a career scientist award in Public Health funded by the NHS Research and Development Programme. The views and opinions expressed in the paper do not necessarily reflect those of the NHS Executive.

Competing interests: None declared.

Ethical approval: The eVALuate trial was approved by the relevant multicentre ethics committee and the local research ethics committee.

1 Vessey MP, Villard-Mackintosh L, McPherson K, Coulter A, Yeates D. The epidemiology of hysterectomy: findings in a large cohort study. Br J Obstet Gynaecol 1992;99:402-7.

Lepine LA, Hillis SD, Marchbanks PA, Koonin LM, Morrow B, Kieke BA, et al. HysterLepine LA, Hillis SD, Marchbanks PA, Koonin LM, Morrow B, Kieke BA, et al. Hyster-

Lowell L, Kessler AA. Laparoscopically assisted vaginal hysterectomy-a suitable Lowell L, Kessler AA. Laparoscopically assisted vaginal hysterect
substitute for abdominal hysterectomy? J Reprod Med 2000;45:738-42.

Schneider A, Merker A, Martin C, Michels W, Krause N. Laparoscopically assisted vaginal hysterectomy as an alternative to abdominal hysterectomy in patients with fibroids. Arch Gynecol Obstet 1997;259:79-85.

5 Chapron C, Fernandez B, Dubuisson JB. Total hysterectomy for benign pathologies: direct costs comparison between laparoscopic and abdominal hysterectomy. Eur J Obstet Gynecol Reprod Biol 2000;89:141-7.

6 Lumsden MA, Twaddle S, Hawthorn R, Traynor I, Gilmore D, Davis J, et al. A randomised comparison and economic evaluation of laparoscopic-assisted hysterectomy and abdominal hysterectomy. Br J Obstet Gynaecol 2000;107:1386-91.

7 Ellstrom M, Ferraz-Nunes J. Hahlin M, Olsson JH. A randomized trial with a cost-consequence analysis after laparoscopic and abdominal hysterectomy. Obstet cost-consequence analys

8 Garry R, Fountain J, Mason S, Hawe J, Napp V, Abbott J, et al. The eVALuate study: two parallel randomised trials, one comparing laparoscopic with abdominal hysterectomy, 
the second comparing laparoscopic with vaginal hysterectomy. BMJ 2004 doi= 10.1136/bmj. $.37958 .696840 .5 \mathrm{~A}$

9 Sculpher MJ, Smith DH, Clayton T, Henderson RA, Buxton MJ, Pocock SJ, et al. Coronary angioplasty versus medical therapy for angina: health service costs based on the second randomised intervention treatment of angina (RITA-2) trial. Eur Heart J 2002;23:1291-300.

10 Booth C. Review body for nursing staff, midwives, health visitors and professions allied to medicine. 17th report. London: Stationery Office, 2000. (Cm 4563.)

11 Gough CB. Review body on doctors' and dentists' remuneration. 29th report. London: Stationery Office, 2000. (Cm 4562.)

12 Netten A, Dennett J, Knight J. Unit costs of health and social care. Canterbury: PSSRU, University of Kent, 2000.

13 Chartered Institute of Public Finance and Accountancy (CIPFA). The health service database 2000. Croydon: CIPFA, 2000.

14 Kind P. The EuroQoL instrument: an index of health-related quality of life. In: Spilker B, ed. Quality of life and pharmacoeconomics in clinical trials. 2nd ed. Philadelphia: Lippincott-Raven, 1996.

15 Dolan P, Gudex C, Kind P, Williams A. A social tariff for Euro Qol:results from a UK general population survey. Centre for Health Economics, University of York: Centre for Health Economics, 1995. (Discussion paper 138.)

16 Matthews JNS, Altman D, Campbell MJ. Analysis of serial measurements in medical research. BMJ 1990;300:230-5.

17 Lin DY, Feuer EJ, Etzioni R, Wax Y. Estimating medical costs from incomplete follow-up data. Biometrics 1997;53:419-34.

18 Willan AR, Lin DY. Incremental net benefit in randomised clinical trials. Stat Med 2001;20:1563-74.

19 Efron B, Tibshirani R. An introduction to the bootstrap. New York: Chapman and Hall, 1993.

20 Barber JA, Thompson SG. Analysis of cost data in randomised controlled trials: an application of the non-parametric bootstrap. Stat Med 2000;19:3219-36.
21 Van Hout BA, Al MJ, Gordon GS, Rutten FFH. Costs, effects and c/e-ratios alongside a clinical trial. Health Econ 1994:3:309-19.

22 Garry R, Fountain J, Braunholtz D, Bridgman S, Brown J, Lilford R, et al. EVALUATE hysterectomy trial. A multicentre randomised trial comparing abdominal, vaginal and laparoscopic methods of hysterectomy. Health Technol Assess (in press).

23 Raftery J. NICE: faster access to modern treatments? Analysis of guidance on health technologies. BMJ 2001;323:1300-3.

24 Sculpher MJ. The role and estimation of productivity costs in economic evaluation. In: Drummond MF, McGuire $\mathrm{AF}$ eds. Theory and practice of economic evaluation in health. Oxford: Oxford University Press, 2001.

(Accepted 10 November 2003)

doi 10.1136/bmj.37942.601331.EE

Centre for Health Economics, University of York, Heslington, York YO10 5DD Mark Sculpher professor

Andrea Manca research fellow

Department of Endo-Gynaecology, University of New South Wales, Royal Hospital for Women, Barker Street, Randwick, NSW 2031, Australia Jason Abbott deputy director

Clinical Trials and Research Unit, University of Leeds, Leeds LS2 9NG

Jayne Fountain medical statistician

Su Mason principal research fellow

School of Women's and Infants' Health, University of Western Australia, King Edward Memorial Hospital, Perth 6008, Australia Ray Garry professor

Correspondence to: M Sculpher mjs23@york.ac.uk 